Arq. Bras. Med. Vet. Zootec., v.59, n.1, p.26-29, 2007

\title{
Avaliação dos reflexos espinhais em cordeiros da raça Suffolk
}

[Evaluation of spinal reflexes in Suffolk lambs]

\author{
L.C.N. Mendes ${ }^{1}$, G.M. Nogueira ${ }^{2}$, A.S. Borges ${ }^{3}$, J.R. Peiró ${ }^{1}$, F.L.F. Feitosa ${ }^{1}$ \\ ${ }^{1}$ Curso de Medicina Veterinária - UNESP \\ Rua Clóvis Pestana, 793 \\ 16050-680 - Araçatuba, SP \\ ${ }^{2}$ Médico veterinário residente - UNESP - Araçatuba, SP \\ ${ }^{3}$ Faculdade de Medicina Veterinária e Zootecnia - UNESP - Botucatu, SP
}

\begin{abstract}
RESUMO
Fez-se a avaliação qualitativa e semiquantitativa dos reflexos espinhais em ovinos, utilizando-se 51 animais da raça Suffolk, machos e fêmeas, entre quatro e cinco meses de idade. Usaram-se os reflexos bilaterais dos membros torácicos, extensor carpo-radial, bicipital, tricipital e flexor, e pélvicos, isquiático, gastrocnêmio, patelar, tibial cranial e flexor, sendo zero indicativo de ausência de reflexo, $1=$ reflexo discreto e $2=$ reflexo evidente. Nos membros torácicos, as melhores respostas foram obtidas no flexor $(99,0 \%)$ e no extensor carpo-radial $(87,3 \%)$, seguidos de valores menos expressivos no bicipital $(11,8 \%)$ e no tricipital $(2,0 \%)$, com grau 2 de avaliação. Nos membros pélvicos, todos os ovinos produziram respostas em grau 2 para o reflexo flexor. Verificam-se também respostas evidentes nos reflexos patelar $(98,0 \%)$ e isquiático $(81,4 \%)$. Apenas $20,6 \%$ dos animais apresentaram resposta evidente ao reflexo tibial cranial, e nenhum ovino respondeu ao reflexo gastrocnêmio de forma satisfatória.
\end{abstract}

Palavras-chave: ovino, reflexo espinhal, exame neurológico

\begin{abstract}
This study was carried out to determine a qualitative and semiquantitative evaluation of the spinal reflexes in 51 both sexes, 4 to 5 months of age Suffolk lambs. Spinal reflexes were evaluated in the thoracic (extensor carpi reflex, biceps reflex, triceps reflex and flexor reflex) and pelvic (ischiatic reflex, gastrocnemius reflex, patellar reflex, cranial tibial reflex and flexor reflex) limbs, bilaterally. Reflexes were standardized, using a 2-point grading scale $(0=$ absense, $1=$ mild reflex, $2=$ evident $)$. Flexor reflex $(99.0 \%)$ and extensor carpi reflex (87.3\%) were the best reflexes elicited in thoracic limbs, while the least evident scores were found in biceps (11.8\%) and triceps reflexes (2.0\%) with grade 2. In pelvic limbs, all of the ovines showed a grade-2 flexor reflex. A high percentage of evident response in patellar (98.0\%) and ischiatic (81.4\%) reflexes were also observed. Only $20.6 \%$ of the animals showed evident response to the cranial tibial reflex but none had a satisfactory response to the gastrocnemius reflex.
\end{abstract}

Keywords: sheep, spinal reflex, neurological examination

\section{INTRODUÇÃO}

Enfermidades que acometem o sistema nervoso são freqüentes em bovinos, ovinos, caprinos e eqüinos, sendo o exame neurológico um passo de fundamental importância para a localização, diagnóstico e prognóstico dessas doenças (Borges et al., 1999).

A neurologia clínica em animais de grande porte não é tão avançada como em humanos e em animais de pequeno porte, entretanto na última

Recebido em 11 de fevereiro de 2005

Aceito em 7 de novembro de 2006

E-mail:1mendes@fmva.unesp.br 
década, consideráveis progressos foram feitos na neurologia eqüina. Parte dessa defasagem devese às falhas dos clínicos de grandes animais em relatar os sinais clínicos observados para subseqüente determinação da localização da lesão (Radostits et al., 2002).

Um exame neurológico completo baseia-se na avaliação do comportamento, do nível de consciência, dos pares de nervos cranianos, das reações posturais e da resposta à estimulação dos reflexos espinhais. Pode-se incluir análises complementares como a do líquido cefalorraquidiano, radiografias simples ou contrastada (mielografia), eletroencefalografia, tomografias computadorizadas e ressonância magnética (Brewer, 1987; Braund, 1994).

Quando comparado aos outros sistemas, o nervoso não é tão acessível ao exame físico, pois não pode ser inspecionado diretamente (com exceção do nervo óptico), auscultado ou palpado, por isso deve ser clinicamente examinado via suas funções (Parker, 1990).

A avaliação racional das funções do sistema nervoso permite verificar se estas estão anormais, determinar as localizações neuroanatômicas de acometimento e formular um diagnóstico diferencial realista, um planejamento terapêutico e um prognóstico (Bagley e Mayhew, 2002). Para tanto deve-se conhecer os padrões funcionais de normalidade.

Reflexos são respostas biológicas involuntárias úteis ao organismo. $\mathrm{O}$ arco reflexo é uma resposta básica após um estímulo, e é por meio de suas várias modalidades (reflexos espinhais, reflexos dos nervos cranianos) que o exame neurológico é efetivado. A estimulação dos reflexos espinhais pode fornecer muitas informações, como por exemplo, se existem ou não lesões em neurônio motor, inferior ou superior, e sua localização na medula espinhal ou até mesmo no encéfalo (Borges et al., 1997; Smith-Maxie, 1997).

Nos animais domésticos, os reflexos freqüentemente testados nos membros torácicos são flexor, bicipital, tricipital e carpo-radial, e nos membros pélvicos, patelar, tibial cranial, gastrocnêmio, isquiático e flexor (Borges et al., 1997).
Apesar da importância mundial da criação de ovinos, poucos são os trabalhos encontrados na literatura que avaliam os reflexos espinhais. Hofmeyr (1978), na tentativa de estabelecer padrões de respostas normais, realizou exame neurológico em 100 ovinos da raça merino e concluiu que os resultados foram insatisfatórios.

Sattler et al. (1998) observaram, em um ovino com encefalomalácia focal simétrica, aumento do tônus muscular nos membros pélvicos, e que os reflexos tríceps e patelar apresentavam-se de normais a aumentados bilateralmente, com diminuição do reflexo de dor.

Anderson et al. (1999), após exame neurológico em neonatos ovinos com doença do neurônio motor inferior, descreveram diminuição dos reflexos flexores com normalidade nos outros reflexos espinhais. Os reflexos flexores também se apresentaram alterados em ovinos com listeriose, isto é, aumentaram em seis e diminuíram ou estavam ausentes em 14 dos 67 animais estudados (Braun et al., 2002).

Os objetivos deste estudo foram avaliar e quantificar a ocorrência dos reflexos espinhais em ovinos jovens, clinicamente sadios.

\section{MATERIAL E MÉTODOS}

Utilizaram-se 51 ovinos da raça Suffolk, com idades entre quatro e cinco meses, clinicamente normais, criados em sistema intensivo (confinamento).

Após contenção em decúbito lateral, sem o auxílio de cordas ou sedação, a avaliação foi realizada a partir do momento em que os animais demonstraram tranqüilidade, representada pelo relaxamento muscular nos quatro membros, e a não tentativa de se colocarem em decúbito esternal ou em posição quadrupedal.

Foram testados, na seguinte ordem, os reflexos bicipital, tricipital, extensor carpo-radial, patelar, isquiático, gastrocnêmio, tibial cranial e flexor.

Os resultados foram obtidos por meio da estimulação dos reflexos nos locais indicados por Mayhew (1989) e De Lahunta (1983), pela percussão com martelo específico, à exceção do reflexo flexor no qual utilizou-se o pinçamento 
do espaço interdigital com pinça hemostática Rochester (na sua primeira ranhura). Os resultados foram documentados após três respostas com a mesma intensidade em testes realizados com aproximadamente 10 segundos de intervalo. Os reflexos flexores, nos membros torácicos e pélvicos, foram testados após a avaliação dos outros reflexos. A quantificação das respostas obedeceu a um padrão numérico, sendo o grau 0 a ausência de reflexo, o grau 1 a presença discreta de reflexos, e o grau 2 a presença evidente do reflexo testado. Para o reflexo flexor, considerou-se somente a intensidade de flexão do membro, e não consideraram a vocalização ou a movimentação da cabeça. Estes resultados foram quantificados em percentuais para cada reflexo.

\section{RESULTADOS E DISCUSSÃO}

No membro torácico, as melhores respostas foram obtidas nos reflexos flexor e extensor carpo-radial, aos quais $99,0 \%$ e $87,3 \%$ dos animais responderam, respectivamente, apresentaram grau 2 de avaliação, seguidos de percentuais menos expressivos nos reflexos bicipital, $11,8 \%$ e tricipital, $2,0 \%$ para o mesmo grau .

No membro pélvico, todos os ovinos produziram respostas em grau 2 para o reflexo flexor. Foram ainda evidenciados altos percentuais de resposta evidente nos reflexos patelar $(98,0 \%)$ e isquiático $(81,4 \%)$. Apenas 20,6\% dos animais apresentaram resposta evidente ao reflexo tibial cranial. Nenhum ovino respondeu ao reflexo gastrocnêmio de forma satisfatória (Tab. 1).

Os resultados deste trabalho diferem dos apresentados por Hofmeyr (1978) que, ao avaliar o reflexo flexor nos membros torácicos e pélvicos de ovinos, observou quase a ausência de resposta em $82 \%$ e $80 \%$, respectivamente. Esse autor utilizou apenas a pressão digital na área interdigital para provocar o estímulo, diferentemente deste trabalho.

Quanto ao reflexo patelar, Hofmeyr (1978) demonstrou que todos os animais respondiam ao estímulo, aspecto constatado neste estudo.
Tabela 1. Freqüência das respostas para cada reflexo espinhal testado nos membros torácicos (T) e pélvicos (P) de cordeiros da raça Sulfolk

\begin{tabular}{llccc}
\hline Membro & Reflexo & Grau 0 & Grau 1 & Grau 2 \\
\hline $\mathrm{T}$ & Bicipital & $64,71 \%$ & $23,53 \%$ & $11,76 \%$ \\
$\mathrm{~T}$ & Tricipital & $90,20 \%$ & $7,84 \%$ & $1,96 \%$ \\
$\mathrm{~T}$ & Extensor carpo-radial & $2,94 \%$ & $9,80 \%$ & $87,26 \%$ \\
$\mathrm{~T}$ & Flexor & $0 \%$ & $0,98 \%$ & $99,02 \%$ \\
$\mathrm{P}$ & Patelar & $0 \%$ & $1,96 \%$ & $98,04 \%$ \\
$\mathrm{P}$ & Isquiático & $4,90 \%$ & $13,73 \%$ & $81,37 \%$ \\
$\mathrm{P}$ & Gastrocnêmio & $97,06 \%$ & $2,94 \%$ & $0 \%$ \\
$\mathrm{P}$ & Tibial cranial & $18,63 \%$ & $60,78 \%$ & $20,59 \%$ \\
$\mathrm{P}$ & Flexor & $0 \%$ & $0 \%$ & $100 \%$ \\
\hline *Grau & 0 ausência de resposta reflexa, grau 1 = presença \\
discreta do reflexo e grau 2 & presença evidente do reflexo.
\end{tabular}

A presença de anormalidades nos reflexos de ovinos com problemas neurológicos, como descrito por Sattler et al. (1998), Anderson et al. (1999) e Braun et al. (2002), ressalta a importância de realização do exame neurológico e a correta interpretação dos resultados. $\mathrm{Na}$ avaliação realizada neste trabalho tem-se a noção semiquantitativa e qualitativa dos reflexos espinhais realizáveis e presentes em ovinos. Os reflexos que mostraram resposta evidente, como os flexores em ambos os membros, o extensor carpo-radial no membro torácico, e os patelar e isquiático no membro pélvico, devem ser utilizados como base do exame neurológico de cordeiros, enquanto que os reflexos que apresentaram resposta pouco evidente, bicipital, tricipital, tibial cranial e gastrocnêmio poderiam ser elicitados em animais com suspeita de lesão do neurônio motor superior, pois sua presença, associada à hiperreflexia nos demais reflexos espinhais, é indicativa desse tipo de lesão. Estes resultados estão de acordo com os de De Lahunta (1983), ao ressaltar que mesmo em cães normais os reflexos bicipital, tricipital, tibial cranial e gastrocnêmio podem estar ausentes.

Diferentemente das descrição feita em cães por De Lahunta (1983), e semelhante ao descrito por Borges (1997) em bezerros, este trabalho ressalta que o reflexo carpo-radial é bastante confiável para ser testado em ovinos.

A escolha de cordeiros para a avaliação e quantificação dos reflexos foi devido ao maior número de casos de problemas neurológicos tétano, mielite ascendente, traumas medulares, abscessos medulares e encefálicos - nessa faixa etária, no hospital veterinário. Contudo, a avaliação em animais adultos também deve ser 
realizada para evitar extrapolações entre espécies ou faixas etárias.

A não resposta a alguns reflexos ou a resposta menos evidente ocorre em diversas espécies, em maior ou menor grau, ressaltando a importância e a necessidade da padronização da estimulação dos reflexos na espécie a ser examinada, para que não ocorra interpretação equivocada que induza ao erro diagnóstico.

\section{CONCLUSÕES}

Os reflexos mais evidentes nos membros torácicos, flexor e o extensor carpo-radial, e nos membros pélvicos, flexor, patelar e o isquiático, são os mais confiáveis para realização do exame neurológico em ovinos da raça e na faixa etária estudadas.

\section{AGRADECIMENTOS}

Ao Sr. Arnaldo Viera Filho (Dindo), da Fazenda Boizendinha, pela cessão dos animais utilizados.

\section{REFERÊNCIAS BIBLIOGRÁFICAS}

ANDERSON, P.D.; PARTON, K.N.; COLLET, M.G. et al. A lower motor neuron disease in newborn rommey lambs. New Zeal. Vet. J., v.47, p.112-114, 1999.

BAGLEY, R.S.; MAYHEW, I.G. Exame clínico do sistema nervoso. In: RADOSTITS, O.M.; MAYHEW, I.G.; HOUSTON, D.M. (Eds.). Exame clínico e diagnóstico em veterinária. Rio de Janeiro: Guanabara Koogan, 2002. cap.19. p.384-422.

BORGES, A.S.; MENDES, L.C.N.; KUCHEMBUCK, M.R.G. Exame neurológico em grandes animais. Parte I: Encéfalo. Rev. Ed. Cont. CRMV-SP, v.2, p.4-16, 1999.
BORGES, A.S.; SAPATERA, A.C.; MENDES, L.C.N. Avaliação dos reflexos espinhais em bezerros. Cien. Rural, v.27, p.613-617, 1997.

BRAUN, U.; STEHLE, C.; EHRENSPERGER, C. Clinical findings and treatment of listeriosis in 67 sheep and goats. Vet. Rec., v.150, p.38-42, 2002.

BRAUND, K.G. Clinical syndromes in veterinary neurology. 2.ed. St Louis: Mosby, 1994. 477p.

BREWER, B.D. Examination of the bovine nervous system. Vet. Clin. N. Am.: Bovine Neurol. Dis., v.3, p.18-27, 1987.

DE LAHUNTA, A. Veterinary neuroanatony and clinical neurology. 2.ed. Philadelphia: Saunders, 1983. 471p.

HOFMEYR, C.F.B. Evaluation of neurological examination of sheep. J. S. Afr. Vet. Assoc., v.49, p.45-48, 1978.

MAYHEW, I.G. Large animal neurology: a handbook for veterinary clinicians. Philadelphia: Lea \& Febiger, 1989. 380p.

OLIVER, J.E. Localization of lesions: the anatomic diagnosis. Progr. Vet. Neurol., v.1, p.28-39, 1990.

PARKER, A.J. Principles of neurologic diagnosis. Progr. Vet. Neurol., v.1, p.11-17, 1990.

RADOSTITS, O.M.; GAY, C.C.; BLOOD, D.C. et al. Clínica veterinária. Um tratado de doenças dos bovinos, ovinos, suínos, caprinos e eqüinos. 9.ed. Rio de Janeiro: Guanabara Koogan, 2002. p.448-492.

SATLER, N.; FECTEAU, G.; DESNOYERS, M. et al. Focal symmetrical encephalomalacia in a 6month-old dorset sheep. Can. Vet. J., v.39, p.434-437, 1998.

SMITH-MAXIE, L. Diseases of the nervous system. In: GREENOUGH, P.R.; WEAVER, A.D. (Eds.). Lameness in cattle. Philadelphia: Saunders, 1997. p.203-218. 